01

\title{
Уравнение огибающей релятивистского электронного пучка, распространяющегося по омическому плазменному каналу при развитии резистивной шланговой неустойчивости
}

\author{
(C) А.С. Мануйлов
}

Санкт-Петербургский государственный университет, 198504 Санкт-Петербург, Россия

e-mail: man06@mail.ru

(Поступило в Редакцию 29 января 2014 г. В окончательной редакции 7 апреля 2016 г.)

С помощью кинетических методов получены уравнения переноса, уравнение вириала, условие динамического равновесия и уравнение огибающей аксиально-симметричного параксиального релятивистского электронного пучка, распространяющегося в омическом плазменном канале в случае развития резистивной шланговой неустойчивости пучка. Получено обобщение уравнения огибающей пучка с учетом указанной неустойчивости.

DOI: $10.21883 /$ JTF.2017.02.44120.820

\section{Введение}

В последние три десятилетия внимание зарубежных, а также отечественных исследователей привлекают вопросы транспортировки релятивистских электронных пучков (РЭП) в плотных и разреженных газоплазменных средах [1-17]. Особый интерес представляет изучение деструктивных процессов, влияющих на динамику РЭП, таких как поперечная дисперсия пучка, разного рода крупномасштабные неустойчивости, эрозия фронтальной части пучка.

Известно, что в результате развития крупномасштабной резистивной неустойчивости с азимутальным числом $\tilde{m}=1$ или так называемой резистивной шланговой неустойчивости (РШН) РЭП, распространяющийся в плотной газоплазменной среде по предварительно созданному плазменному каналу, может быть смещен относительно оси указанного канала, вдоль которого он распространяется [11-17]. При этом ось симметрии коллективного самосогласованного электромагнитного поля в силу резистивности газоплазменной фоновой среды будет отставать от оси пучка. В результате нарушится самофокусировка РЭП и он будет испытывать дополнительную поперечную дисперсию. В связи с вышесказанным значительный интерес представляет задача формулировки уравнения огибающей РЭП при развитии РШН.

\section{Постановка задачи}

Рассмотрим параксиальный моноэнергетический РЭП, распространяющийся в плотной газоплазменной среде вдоль предварительно созданного искусственного омического плазменного канала. Будем считать, что радиальный профиль плотности обратного равновесного плазменного тока $J_{p z 0}\left(\mathbf{r}_{\perp}\right)$ совпадает с соответствующим профилем плотности тока пучка $J_{b z 0}\left(\mathbf{r}_{\perp}\right)$, так, что выполнено соотношение

$$
\alpha_{m}=-\frac{J_{p z 0}\left(\mathbf{r}_{\perp}\right)}{J_{b z 0}\left(\mathbf{r}_{\perp}\right)},
$$

где $\alpha_{m}$ - коэффициент магнитной (токовой) нейтрализации, который в данном случае не зависит от поперечного радиус-вектора $\mathbf{r}_{\perp}$. Далее будем полагать, что имеет место полная зарядовая нейтрализация. Кроме того, предположим, что цилиндрическая система координат $(r, \theta, z)$ связана в рассматриваемой задаче не с плазменным каналом, а с центром смещенного пучка (для простоты здесь предполагается, что РЭП смещается без изгиба, так что ось симметрии пучка остается прямой). Кроме того, будем считать, что внешнее продольное магнитное поле отсутствует $\left(B_{z 0}=0\right)$.

Смещение оси канала относительно оси пучка обозначим как $Y$, амплитуду отклонения оси симметрии самосогласованного коллективного электромагнитного поля системы плазма-пучок относительно оси плазменного канала через $D$. Тогда отклонение оси коллективного поля относительно оси пучка

$$
\mathbf{D}^{*}=\mathbf{Y}-\mathbf{D} \text {. }
$$

Будем считать, что

$$
\frac{Y}{R_{b}} \gg 1, \quad \frac{D}{R_{b}} \ll 1,
$$

где $R_{b}$ - характерный радиус пучка. Последнее условие означает, что РЭП находится на линейной стадии развития РШН [11-17]. В этой ситуации можно считать, что искажение радиального профиля плотности тока пучка $J_{b z 0}\left(\mathbf{r}_{\perp}\right)$ несущественно $[11,12]$.

В этом случае уравнения переноса, полученные из кинетического уравнения, сформулированного в $[7,8]$, сохраняют свой вид при условии следующей замены 
потенциалов:

$$
A_{z}^{(b)}\left(\mathbf{r}_{\perp}\right)+A_{z}^{(p)}\left(\mathbf{r}_{\perp}\right) \rightarrow A_{z}^{*}\left(\mathbf{r}_{\perp}\right),
$$

где $A_{z}^{*}\left(\mathbf{r}_{\perp}\right)-z$-компонента вектор-потенциала, созданного смещенным относительно РЭП коллективным электромагнитным полем. Как отмечалось выше, здесь мы рассматриваем так называемую „жесткую“ модель РШН [11], в которой предполагается, что при боковом смещении радиальные конфигурации плотности тока пучка, потенциала $A_{z}$ и омической проводимости плазменного канала $\sigma$ не меняются. В силу этого предположения считаем, что $A_{z}^{*}\left(\mathbf{r}_{\perp}\right)=A_{z 0}\left(\left|\mathbf{S}^{*}\right|\right)$, где $\mathbf{S}^{*}=\mathbf{r}_{\perp}-\mathbf{D}^{*}$, а $\mathbf{D}^{*}$ определено в (2), $A_{z 0}\left(\mathbf{r}_{\perp}\right)-z-$ компонента векторного потенциала коллективного электромагнитного поля системы плазма-пучок при отсутствии смещения плазменного канала относительно пучка.

Тогда имеем уравнения переноса

$$
\begin{gathered}
\frac{\partial \chi_{b}}{\partial t}+\nabla_{\perp}\left(\chi_{b} \frac{\widetilde{\mathbf{p}_{\perp}}}{\gamma m}\right)=0, \\
\frac{\partial}{\partial t}\left(\chi_{b} \tilde{\mathbf{p}}_{\perp}\right)+\nabla_{\perp}\left(\chi_{b} \frac{\widetilde{\mathbf{p}_{\perp} \mathbf{p}_{\perp}}}{\gamma m}\right)-\chi_{b} e \beta \nabla_{\perp} A_{z}^{*}=0, \\
\frac{\partial}{\partial t}\left(\chi_{b} \frac{\widetilde{p_{\perp}^{2}}}{2 m \gamma}\right)+\frac{1}{\gamma} \frac{d \gamma}{d t} \frac{\chi_{b} \widetilde{p_{\perp}^{2}}}{2 m \gamma}+\nabla_{\perp}\left(\frac{\chi_{b} \widetilde{p_{\perp}^{2}}}{2 m^{2} \gamma^{2}}\right) \\
-e \beta \nabla_{\perp} A_{z}^{*}\left(\frac{\chi_{b} \tilde{\mathbf{p}}_{\perp}}{\gamma m}\right)=\chi_{b} S,
\end{gathered}
$$

где $t-$ время, $\chi_{b}\left(\mathbf{r}_{\perp}, t\right)-$ нормированная к единице плотность частиц пучка в сегменте $S^{\tau}, \nabla_{\perp} \equiv \nabla_{\mathbf{r}_{\perp}} e-$ заряд электрона, $m-$ его масса, $\gamma-$ лоренц-фактор частиц пучка, $\beta=v_{z} / c$ - отношение продольной компоненты скорости электронов пучка к скорости света $c$, $S$ - скорость закачки энергии из продольного движения частиц пучка в поперечное за счет многократного кулоновского рассеяния указанных частиц на атомах и молекулах фоновой газоплазменной среды,

$$
\tilde{\mathbf{p}}_{\perp}\left(\mathbf{r}_{\perp}, t\right)=\frac{1}{\chi_{b}} \int \mathbf{p}_{\perp} f_{b}^{\tau} d \mathbf{p}_{\perp}
$$

- средний поперечный импульс,

$$
\begin{gathered}
\widetilde{\mathbf{p}_{\perp} \mathbf{p}_{\perp}}\left(\mathbf{r}_{\perp}, t\right)=\frac{1}{\chi_{b}} \int \mathbf{p}_{\perp} \mathbf{p}_{\perp} f_{b}^{\tau} d \mathbf{p}_{\perp}, \\
\widetilde{p_{\perp}^{2}}\left(\mathbf{r}_{\perp}, t\right)=\frac{1}{\chi_{b}} \int p_{\perp}^{2} f_{b}^{\tau} d \mathbf{p}_{\perp}, \\
\widetilde{\mathbf{p}_{\perp} p_{\perp}^{2}}\left(\mathbf{r}_{\perp}, t\right)=\frac{1}{\chi_{b}} \int \mathbf{p}_{\perp} p_{\perp}^{2} f_{b}^{\tau} d \mathbf{p}_{\perp} .
\end{gathered}
$$

Здесь $f_{b}^{\tau}\left(\mathbf{r}_{\perp}, \mathbf{p}_{\perp}, t\right)-$ функция распределения электронов пучка в поперечном сегменте $S^{\tau}$ по поперечным координатам $\mathbf{r}_{\perp}$ и импульсам $\mathbf{p}_{\perp}$.

В силу того что $\tilde{\mathbf{p}}_{\perp} / m \gamma=\tilde{\mathbf{v}}_{\perp}$ (где $\tilde{\mathbf{v}}_{\perp}-$ средняя поперечная скорость частиц пучка), уравнение представляет собой обычное уравнение непрерывности, выражающее закон сохранения числа частиц рассматриваемого сегмента пучка.

Уравнение (6) может быть записано в виде

$$
\left(\frac{\partial}{\partial t}+\tilde{\mathbf{v}}_{\perp} \nabla_{\perp}\right) \tilde{\mathbf{p}}_{\perp}=-\frac{\nabla_{\perp} \widetilde{\mathbf{P}}_{\perp}}{\chi_{b}}-e\left[\mathbf{E}_{\perp}^{\mathrm{eff}}+\frac{1}{c}\left(\mathbf{v}_{\perp} \times \mathbf{B}_{z 0}\right)\right],
$$

где $\mathbf{E}_{\perp}^{\mathrm{eff}}=\beta \nabla_{\perp} A_{z}^{*}-$ поперечная компонента эффективного электрического поля,

$$
\widetilde{\widetilde{P}}_{\perp}=\int\left(\mathbf{p}_{\perp}-\tilde{\mathbf{p}}_{\perp}\right)\left(\mathbf{v}_{\perp}-\tilde{\mathbf{v}}_{\perp}\right) f_{b}^{\tau} d \mathbf{p}_{\perp}
$$

— тензор напряжений.

Третий член в левой части уравнения энергии (7) характеризует скорость изменения средней энергии поперечного движения частиц сегмента пучка $S^{\tau}$, связанного с наличием потока энергии с плотностью

$$
\mathbf{R}_{0}=\frac{\chi_{b} \widetilde{\mathbf{p}_{\perp} p_{\perp}^{2}}}{2 m^{2} \gamma^{2}}=\frac{\widetilde{\chi_{b} \mathbf{v}_{\perp} p_{\perp}^{2}}}{2 m \gamma} .
$$

Четвертый член в левой части (7) может быть записан в виде

$$
-\frac{e \beta \chi_{b} \tilde{\mathbf{p}}_{\perp}}{m \gamma} \nabla_{\perp} A_{z}^{*}=\mathbf{J}_{\perp} \mathbf{E}_{\perp}^{\mathrm{eff}},
$$

где $\mathbf{J}_{\perp}=-e \chi_{b} \tilde{\mathbf{p}}_{\perp} / m \gamma=-e \chi_{b} \tilde{\mathbf{v}}_{\perp}$ и $\mathbf{E}_{\perp}^{\mathrm{eff}}$ определено выше.

Как видно из (15), этот член характеризует скорость изменения энергии поперечного движения, обусловленного работой сил, действующих на частицы пучка со стороны самосогласованного коллективного электромагнитного поля.

Наконец, второй член в левой части (7) и $\chi_{b} S$ в правой части (7) характеризуют соответственно скорости изменения энергии поперечного движения, вызываемого неупругими (член, пропорциональный $d \gamma / d t$, в левой части (7)) и упругими столкновениями частиц пучка с частицами газоплазменной среды.

\section{Уравнение вириала для РЭП, смещенного из омического плазменного канала. Условие динамического равновесия}

Умножим уравнение импульса (6) скалярно на $\mathbf{r}_{\perp}$ и проинтегрируем полученное выражение по пространству поперечных координат. После ряда преобразований с учетом (4) получим

$$
\frac{d}{d t}\left(\frac{\gamma m}{4} \frac{d}{d t} \mathscr{R}^{2}\right)=2 E_{\perp}+e \beta \int \chi_{b} \mathbf{r}_{\perp} \nabla_{\perp} A_{z}^{*}\left(\mathbf{r}_{\perp}\right) d \mathbf{r}_{\perp},
$$

где

$$
E_{\perp}=\int \chi_{b} \frac{p_{\perp}^{2}}{2 m \gamma} d \mathbf{r}_{\perp}, \quad \mathscr{R}^{2}=2 \int \chi_{b} r_{\perp}^{2} d \mathbf{r}_{\perp}
$$

- соответственно средняя кинетическая энергия поперечного движения частиц сегмента пучка и среднеквадратичный радиус сегмента пучка. 
Обратимся далее к вычислению второго слагаемого в правой части (16). С учетом того что $\nabla_{\perp} A_{z 0}\left(S^{*}\right) \mathbf{r}_{\perp}=r \partial A_{z 0}\left(S^{*}\right) / \partial r$, где $S^{*}=\left|\mathbf{S}^{*}\right|$, после разложения функции $A_{z 0}\left(S^{*}\right)$ в ряд Тейлора по степеням $D^{*}$ до членов второго порядка малости имеем

$$
\begin{aligned}
\Phi_{1} \equiv & e \beta \int \chi_{b}\left(\mathbf{r}_{\perp}\right) \mathbf{r}_{\perp} \nabla_{\perp} A_{z}^{*}\left(\mathbf{r}_{\perp}\right) d \mathbf{r}_{\perp}=\frac{e \beta}{I_{b}} \int_{0}^{\infty} r d r \\
& \times \int_{0}^{2 \pi} J_{b z 0}(r) r \frac{\partial}{\partial r}\left\{-D^{*} \frac{\partial A_{z 0}(r)}{\partial r} \cos (\theta)+A_{z 0}(r)\right. \\
& \left.+\frac{\left(D^{*}\right)^{2}}{2}\left[\cos ^{2}(\theta) \frac{\partial^{2} A_{z 0}(r)}{\partial r^{2}}+\frac{\sin ^{2}(\theta)}{r} \frac{\partial A_{z 0}(r)}{\partial r}\right]\right\} d \theta,
\end{aligned}
$$

где $\chi_{b}\left(\mathbf{r}_{\perp}\right)=J_{b z 0}\left(\mathbf{r}_{\perp}\right) / I_{b}, J_{b z 0}\left(\mathbf{r}_{\perp}\right)-z$-компонента плотности равновесного тока пучка.

Далее заметим, что $\int_{0}^{2 \pi} \cos (\theta) d \theta=0$ и вклад в выражение (18) дают только члены порядка единицы и $\left(D^{*}\right)^{2}$. Тогда получим

$$
\begin{gathered}
\Psi \equiv e \beta \int_{0}^{\infty} r^{2} d r \int_{0}^{2 \pi} \frac{J_{b z 0}(r)}{I_{b}} \frac{\partial A_{z 0}(r)}{\partial r} d \theta=-\frac{e \beta}{c}\left(1-\alpha_{m}\right) I_{b} \\
\Phi_{1}=\frac{e \beta \pi}{I_{b}} \frac{\left(D^{*}\right)^{2}}{2} \int_{0}^{\infty} r^{2} J_{b z 0}(r) \frac{\partial}{\partial r} \\
\quad \times\left\{\frac{\partial^{2} A_{z 0}(r)}{\partial r^{2}}+\frac{1}{r} \frac{\partial A_{z 0}(r)}{\partial r}\right\} d r+\Psi
\end{gathered}
$$

С другой стороны, очевидно, что

$$
\frac{\partial^{2} A_{z 0}}{\partial r^{2}}+\frac{1}{r} \frac{\partial A_{z 0}}{\partial r}=\frac{1}{r} \frac{\partial}{\partial r}\left(r \frac{\partial A_{z 0}}{\partial r}\right) .
$$

Кроме того, уравнение Ампера для квазистационарных параксиальных РЭП имеет простой вид

$$
\frac{1}{r} \frac{\partial}{\partial r}\left(r \frac{\partial A_{z 0}}{\partial r}\right)=-\frac{4 \pi}{c}\left(1-\alpha_{m}\right) J_{b z 0},
$$

где $\alpha_{m}-$ коэффициент токовой (магнитной) нейтрализации, определенный в (1).

С учетом (21) и (22) выражение (20) может быть записано как

$$
\Phi_{1}=k_{S}^{2}\left(1-\alpha_{m}\right) \frac{(\beta c)^{2}}{2} m \gamma\left(D^{*}\right)^{2}-\frac{e \beta}{c}\left(1-\alpha_{m}\right) I_{b},
$$

где

$$
k_{S}^{2}=\frac{4 \pi^{2} I_{b}}{I_{A}} \int_{0}^{\infty} r \frac{\left[J_{b z 0}(r)\right]^{2}}{I_{0}^{2}} d r
$$

- квадрат характерного волнового числа колебаний РЭП при развитии РШН, $I_{A}$ - предельный ток Альфвена.
Подставляя (23) в уравнение (16), получим

$$
\begin{aligned}
\frac{d}{d t}\left(\frac{\gamma m}{4} \frac{d \mathscr{R}^{2}}{d t}\right)= & 2 E_{\perp}+k_{s}^{2}\left(1-\alpha_{m}\right) \frac{(\beta c)^{2} m \mathcal{\gamma}}{2}\left(D^{*}\right)^{2} \\
& -\frac{e \beta}{c}\left(1-\alpha_{m}\right) I_{b} .
\end{aligned}
$$

Вводя в рассмотрение эффективную температуру Беннета $T_{B}=I_{b} e \beta /(2 c)=m \gamma v_{z}^{2} I_{b} /\left(2 I_{A}\right)\left(I_{b}-\right.$ полный ток пучка, $I_{A}=\beta \gamma m c^{3} / e-$ предельный ток Альфвена, $v_{z}-$ аксиальная компонента скорости частиц пучка), запишем (25) в виде

$$
\begin{aligned}
\frac{d}{d t}\left(\frac{\gamma m}{4} \frac{d \mathscr{R}^{2}}{d t}\right)= & 2\left[E_{\perp}-\left(1-\alpha_{m}\right) T_{B}\right] \\
& +T_{B}\left(1-\alpha_{m}\right) \tilde{k}_{S}^{2}\left(D^{*}\right)^{2}
\end{aligned}
$$

где

$$
\tilde{k}_{S}^{2} \equiv \frac{I_{A}}{I_{b}} k_{S}^{2}=4 \pi^{2} \int_{0}^{\infty} r \frac{\left[J_{b z 0}(r)\right]^{2}}{I_{b}^{2}} d r .
$$

Из (26) нетрудно получить уравнение вириала

$$
E_{\perp}-\frac{d}{d t}\left(\frac{m \gamma}{8} \frac{d \mathscr{R}^{2}}{d t}\right)=V,
$$

где средний вириал определяется в виде

$$
V=-\frac{e \beta}{2} \int \chi_{b}\left(\mathbf{r}_{\perp}\right) \mathbf{r}_{\perp} \nabla_{\perp} A_{z}^{*}\left(\mathbf{r}_{\perp}\right) d \mathbf{r}_{\perp} .
$$

Согласно (18), (19), (23) и (29), имеем

$$
V=-\frac{\Phi_{1}}{2}=-k_{S}^{2} \lambda_{m} \frac{(\beta c)^{2}}{4} m \gamma\left(D^{*}\right)^{2}+\frac{e \beta}{2 c} \lambda_{m} I_{b},
$$

где $\lambda_{m}=1-\alpha_{m}$. Выражая далее $V$ через $T_{B}$, находим

$$
V=T_{B} \lambda_{m}-T_{B} \lambda_{m} \frac{k_{S}^{2}}{2}\left(D^{*}\right)^{2} .
$$

Полагая в (26) производную $d \mathscr{R}^{2} / d t=0$, получим необходимое условие динамического равновесия рассматриваемого сегмента пучка

$$
E_{\perp}=V=T_{B} \lambda_{m}\left[1-\frac{k_{S}^{2}}{2}\left(D^{*}\right)^{2}\right] .
$$

Равенство (32) является обобщением известного условия равновесия Беннета [7] на случай резистивного отставания оси симметрии полевой структуры от оси пучка в случае развития РШН РЭП.

\section{Уравнение для средней полной поперечной энергии частиц РЭП, смещенного из омического плазменного канала}

Рассмотрим полную энергию частиц сегмента пучка $\Psi$, которую определим как сумму средней кинетической энергии поперечного движения $E_{\perp}$ и средней 
потенциальной энергии частиц в эффективном коллективном электрическом поле, которое обозначим как $\mathbf{E}_{\perp}^{\mathrm{eff}}=-\nabla_{\perp} A_{z}^{*}$. Тогда имеем

$$
\Psi=E_{\perp}+\Lambda_{\beta}^{*},
$$

где

$$
\Lambda_{\beta}^{*}=-\frac{1}{2} \int \chi_{b}\left(\mathbf{r}_{\perp}\right) e \beta A_{z}^{*}\left(\mathbf{r}_{\perp}\right) d \mathbf{r}_{\perp}
$$

и, кроме того, $A_{z}^{*}\left(\mathbf{r}_{\perp}\right)=A_{z 0}\left(\mathbf{r}_{\perp}-\mathbf{D}^{*}\right)$.

Чтобы сформулировать уравнение для средней поперечной энергии частиц пучка, продифференцируем (33) по времени. После ряда преобразований

$$
\begin{aligned}
& \frac{d \Psi}{d t}=\frac{d E_{\perp}}{d t}+\frac{d \Lambda_{\beta}^{*}}{d t}=-\frac{E_{\perp}}{\gamma} \frac{d \gamma}{d t}+\frac{\Lambda_{\beta}^{*}}{e \beta I_{n}} \frac{d}{d t}\left(e \beta I_{n}\right) \\
& +\int \chi_{b} S d \mathbf{r}_{\perp}+e \beta I_{n} \int\left\{\frac{\chi_{b} \mathbf{p}_{\perp}}{\gamma m} \nabla_{\perp}\left(\frac{A_{z}^{*}}{I_{n}}\right)-\frac{\partial}{\partial t}\left(\frac{\chi_{b} A_{z}^{*}}{2 I_{n}}\right)\right\} d \mathbf{r}_{\perp},
\end{aligned}
$$

где $I_{n}=I_{p}+I_{b}-$ полный ток системы плазма-пучок. Очевидно, что последнее слагаемое в (35) будет зависеть от величины $D^{*}$. Чтобы определить указанную зависимость, рассмотрим выражение

$$
\mathscr{I}_{1} \equiv \int\left\{\frac{\chi_{b} \mathbf{p}_{\perp}}{\gamma m} \nabla_{\perp}\left(\frac{A_{z}^{*}}{I_{n}}\right)-\frac{\partial}{\partial t}\left(\frac{\chi_{b} A_{z}^{*}}{2 I_{n}}\right)\right\} d \mathbf{r}_{\perp} .
$$

Разложим потенциал $A_{z}^{*}\left(\mathbf{r}_{\perp}\right)=A_{z 0}\left(\mathbf{r}_{\perp}-\mathbf{D}^{*}\right)$ в ряд Тейлора в окрестности радиус-вектора $\mathbf{r}_{\perp}$ и ограничимся членами второго порядка по $D^{*}$. Тогда получим

$$
\begin{aligned}
& A_{z}^{*}\left(\mathbf{r}_{\perp}\right)=A_{z 0}(r)-D^{*} \frac{\partial A_{z 0}(r)}{\partial r} \cos (\theta) \\
& +\frac{\left(D^{*}\right)^{2}}{2}\left[\cos ^{2}(\theta) \frac{\partial^{2} A_{z 0}(r)}{\partial r^{2}}+\frac{\sin ^{2}(\theta)}{r} \frac{\partial A_{z 0}(r)}{\partial r}\right]+\ldots
\end{aligned}
$$

Подставляя (37) в (36), имеем

$$
\begin{aligned}
\mathscr{Y}_{1}= & \frac{\pi}{2} \int_{0}^{\infty} r\left\{\frac{\left(D^{*}\right)^{2}}{I_{n}}\left(\frac{\partial^{2} A_{z 0}}{\partial r^{2}}+\frac{1}{r} \frac{\partial A_{z 0}}{\partial r}\right) \frac{\partial \chi_{b}}{\partial t}\right. \\
& \left.-\chi_{b} \frac{\partial}{\partial t}\left[\frac{\left(D^{*}\right)^{2}}{I_{n}}\left(\frac{\partial^{2} A_{z 0}}{\partial r^{2}}+\frac{1}{r} \frac{\partial A_{z 0}}{\partial r}\right)\right]\right\} d r .
\end{aligned}
$$

В силу тождества (21) из (38) следует

$$
\begin{aligned}
\mathscr{I}_{1}= & \frac{\pi}{4} \int_{0}^{\infty} r\left\{\frac{\left(D^{*}\right)^{2}}{I_{n}}\left[\frac{1}{r} \frac{\partial}{\partial r}\left(r \frac{\partial A_{z 0}}{\partial r}\right)\right] \frac{\partial \chi_{b}}{\partial t}\right. \\
& \left.-\chi_{b} \frac{\partial}{\partial t}\left[\frac{\left(D^{*}\right)^{2}}{I_{n}} \frac{1}{r} \frac{\partial}{\partial t}\left(r \frac{\partial A_{z 0}}{\partial r}\right)\right]\right\} d r .
\end{aligned}
$$

С учетом уравнения (22) и соотношения $\chi_{b}=J_{b z 0} / I_{b}$ после ряда преобразований получим

$$
\begin{aligned}
\mathscr{I}_{1} & =\frac{\pi^{2}}{c} \frac{\partial\left(D^{*}\right)^{2}}{\partial t} \int_{0}^{\infty} r \frac{\left[I_{b z} 0(r)\right]^{2}}{I_{b}^{2}} d r \\
& =\frac{1}{e \beta I_{n}} \frac{k_{S}^{2}}{2} \lambda_{m} v_{z}^{2} m \gamma \frac{\partial\left(D^{*}\right)^{2}}{\partial t} .
\end{aligned}
$$

Тогда из (35) находим уравнение для средней полной поперечной энергии частиц пучка

$$
\begin{aligned}
\frac{d E_{\perp}}{d t}= & \int \chi_{b} S d \mathbf{r}_{\perp}-\frac{E_{\perp}}{\gamma} \frac{d \gamma}{d t}-\Lambda_{\beta}^{*} \frac{d}{d t}\left[\ln \left(\frac{\Lambda_{\beta}^{*}}{e \beta I_{n}}\right)\right] \\
& +\frac{k_{S}^{2} \lambda_{m}}{4} v_{z}^{2} m \gamma \frac{\partial\left(D^{*}\right)^{2}}{\partial t} .
\end{aligned}
$$

Используя соотношения (37) и (22), можно получить выражение для $\Lambda_{\beta}^{*}$ в форме

$$
\Lambda_{\beta}^{*}=\Lambda_{\beta 0}+k_{S}^{2} \lambda_{m} \frac{m \gamma(\beta c)^{2}}{4}\left(D^{*}\right)^{2} .
$$

Тогда (35) принимает вид

$$
\begin{aligned}
\frac{d E_{\perp}}{d t}=\int \chi_{b} S d \mathbf{r}_{\perp} & -\frac{E_{\perp}}{\gamma} \frac{d \gamma}{d t}-\left[\frac{d \Lambda_{\beta 0}}{d t}-\frac{\Lambda_{\beta 0}}{\lambda_{m} T_{B}} \frac{d\left(\lambda_{m} T_{B}\right)}{d t}\right] \\
& -\frac{\lambda_{m} T_{B}}{2}\left(D^{*}\right)^{2} \frac{d \tilde{k}_{S}^{2}}{d t}
\end{aligned}
$$

где $\lambda_{m}=1 \alpha_{m}$, величина $k_{S}^{2}$ определена в (27).

Нетрудно видеть, что (43) можно записать в виде

$$
\frac{d E_{\perp}}{d t}=\left(\frac{d E}{d t}\right)_{\mathrm{enc}}+\left(\frac{d E}{d t}\right)_{\mathrm{los}}+\left(\frac{d E}{d t}\right)_{\beta}+\left(\frac{d E}{d t}\right)_{\text {hose }},
$$

где

$$
\begin{gathered}
\left(\frac{d E}{d t}\right)_{\mathrm{enc}}=\int \chi_{b} S d \mathbf{r}_{\perp}, \\
\left(\frac{d E}{d t}\right)_{\mathrm{los}}=-\frac{E_{\perp}}{\gamma} \frac{d \gamma}{d t}, \\
\left(\frac{d E}{d t}\right)_{\beta}=-\Lambda_{\beta 0} \frac{d}{d t}\left[\ln \left(\frac{\Lambda_{\beta 0}}{e \beta I_{n}}\right)\right], \\
\left(\frac{d E}{d t}\right)_{\text {hose }}=-\frac{\lambda_{m} T_{B}}{2}\left(D^{*}\right)^{2} \frac{d \tilde{k}_{S}^{2}}{d t}
\end{gathered}
$$

- соответственно скорости изменения средней поперечной кинетической энергии частиц сегмента РЭП за счет упругих и неупругих столкновений частиц пучка с частицами фоновой газоплазменной среды (формулы (45) и (46)), (47) - соответствующая скорость за счет работы сил, действующих на частицы пучка со стороны самосогласованного эффективного коллективного электрического поля $\mathbf{E}_{\perp}^{\mathrm{eff}}=-\nabla_{\perp} A_{z 0}$, а (48) - скорость изменения $E_{\perp}$ за счет вариации во времени квадрата расстояния между центром пучка и осью симметрии коллективного поля. 
Можно отметить, что из уравнения (32) видно, что слагаемое, равное $-\left(T_{B} \lambda_{m} / 2\right) \tilde{k}_{S}^{2}\left(D^{*}\right)^{2}$, характеризует ослабление средней кинетической энергии поперечного движения частиц рассматриваемого сегмента пучка за счет дефокусировки пучка (отставания оси симметрии поля от оси симметрии коллективного азимутального магнитного поля. Поэтому физический смысл величины (48) - это скорость изменения средней кинетической энергии электронов пучка в рассматриваемом сегменте РЭП в результате наличия дополнительной дефокусировки пучка за счет указанного отставания (запаздывания) оси симметрии полевой структуры от оси смещенного пучка. Можно определить величину $\tilde{k}_{S}^{2}$, например, для беннетовского профиля плотности тока РЭП: $\tilde{k}_{s}^{2}=2 /\left(3 R_{b}^{2}\right)$, где $R_{b}^{2}-$ квадрат характерного радиуса пучка. Тогда

$$
\frac{d \tilde{k}_{S}^{2}}{d t}=-\frac{4}{3 R_{b}^{3}} \frac{d R_{b}}{d t} .
$$

Предполагая, что слагаемые (45) и (46) доминируют в процессе распространения пучка и его среднеквадратичный радиус, а следовательно, и характерный радиус нарастает (т.е. $d R_{b} / d t>0$ ), мы получим, что слагаемое (48) больше нуля и работает аналогично процессу рассеяния (слагаемое (45)) и потерям энергии (слагаемое (46)). А именно работает на дефокусировку (расширение) РЭП.

\section{Уравнение для среднеквадратичного радиуса сегмента пучка (уравнение огибающей пучка)}

Определим вид уравнения для среднеквадратичного радиуса сегмента РЭП или так называемое уравнение огибающей параксиального аксиально-симметричного пучка при наличии эффектов многократного упругого рассеяния на частицах фонового газа, неламинарности пучка в ситуации, когда РЭП смещен из омического плазменного канала при развитии РШН.

С этой целью обе части уравнения для средней полной поперечной энергии (43) умножим на лоренц-фактор $\gamma$. Тогда получим

$$
\begin{aligned}
\frac{d\left(\gamma E_{\perp}\right)}{d t}= & \gamma \int \chi_{b} S d \mathbf{r}_{\perp}+\gamma \Lambda_{\beta 0} \frac{d}{d t}\left[\ln \left(\frac{\lambda_{m} T_{B}}{\Lambda_{\beta 0}}\right)\right] \\
& -\frac{\lambda_{m} T_{B} \gamma}{2}\left(D^{*}\right)^{2}\left(\frac{d \tilde{k}_{S}^{2}}{d t}\right) .
\end{aligned}
$$

Рассмотрим теперь уравнение вириала в форме (26). После умножения (26) на $\gamma / 2$ и дифференцирования полученного уравнения по $t$ имеем

$$
\begin{aligned}
\frac{d\left(\gamma E_{\perp}\right)}{d t}= & \frac{1}{2} \frac{d}{d t}\left\{\gamma \frac{d}{d t}\left[\frac{\gamma m}{4} \frac{d \mathscr{R}^{2}}{d t}\right]\right\}+\frac{d}{d t}\left(\gamma \lambda_{n} T_{B}\right) \\
& -\frac{1}{2} \frac{d}{d t}\left(\gamma \lambda_{m} T_{B}\left(D^{*}\right)^{2} \tilde{k}_{S}^{2}\right) .
\end{aligned}
$$

Приравнивая правые части (49) и (50), приходим к уравнению

$$
\begin{aligned}
& \frac{1}{2} \frac{d}{d t}\left\{\gamma \frac{d}{d t}\left[\frac{\gamma m}{4} \frac{d \mathscr{R}^{2}}{d t}\right]\right\}+\frac{d}{d t}\left(\gamma \lambda_{m} T_{B}\right)-\frac{1}{2} \frac{d}{d t} \\
& \quad \times\left(\gamma \lambda_{m} T_{B}\left(D^{*}\right)^{2} \tilde{k}_{S}^{2}\right)=\gamma \int \chi_{b} S d \mathbf{r}_{\perp}+\gamma \Lambda_{\beta 0} \frac{d}{d t} \\
& \quad \times\left[\ln \left(\frac{\lambda_{m} T_{B}}{\Lambda_{\beta 0}}\right)\right]-\frac{\lambda_{m} T_{B} \gamma}{2}\left(D^{*}\right)^{2}\left(\frac{d \tilde{k}_{S}^{2}}{d t}\right) .
\end{aligned}
$$

Откуда находим

$$
\begin{aligned}
& \frac{1}{2} \frac{d}{d t}\left\{\gamma \frac{d}{d t}\left[\frac{\gamma m}{4} \frac{d \mathscr{R}^{2}}{d t}\right]\right\}=\gamma \Lambda_{\beta 0} \frac{d}{d t}\left[\ln \left(\frac{\lambda_{m} T_{B}}{\Lambda_{\beta 0}}\right)\right] \\
& \quad+\gamma \int \chi_{b} S d \mathbf{r}_{\perp}-\frac{d}{d t}\left(\gamma \lambda_{m} T_{B}\right)+\frac{\tilde{k}_{S}^{2}}{2} \frac{d}{d t}\left(\gamma \lambda_{m} T_{B}\left(D^{*}\right)^{2}\right) .
\end{aligned}
$$

Далее воспользуемся следующим тождеством:

$$
\begin{aligned}
\mathscr{R}^{2} \frac{d}{d t}\left\{\gamma \frac{d}{d t}\left[\frac{\gamma m}{4} \frac{d \mathscr{R}^{2}}{d t}\right]\right\} \equiv & \frac{d}{d t}\left[\frac { m } { 2 } \left\{\gamma^{2} \mathscr{R}^{3} \frac{d^{2} \mathscr{R}}{d t^{2}}\right.\right. \\
& \left.\left.+\gamma \frac{d \gamma}{d t} \mathscr{R}^{3} \frac{d \mathscr{R}}{d t}\right\}\right] .
\end{aligned}
$$

Тогда уравнение (52) сводится к виду

$$
\begin{aligned}
& \frac{d}{d t}\left(\gamma^{2} \mathscr{R}^{3} \frac{d^{2} \mathscr{R}}{d t^{2}}+\gamma \frac{d \gamma}{d t} \mathscr{R}^{3} \frac{d \mathscr{R}}{d t}\right)=\frac{4 \mathscr{R}^{2} \gamma}{m} \\
& \times\left\{\Lambda_{\beta 0} \frac{d}{d t}\left[\ln \left(\frac{\lambda_{m} T_{B}}{\Lambda_{\beta 0}}\right)\right]+\int \chi_{b} S d \mathbf{r}_{\perp}-\frac{1}{\gamma} \frac{d}{d t}\left(\gamma \lambda_{m} T_{B}\right)\right. \\
& \left.+\frac{\tilde{k}_{S}^{2}}{2 \gamma} \frac{d}{d t}\left(\gamma \lambda_{m} T_{B}\left(D^{*}\right)^{2}\right)\right\} .
\end{aligned}
$$

Рассмотрим далее выражение

$$
\varphi_{1} \equiv \int_{\tau}^{t} \frac{4 \mathscr{R}^{2} \gamma}{m}\left[-\frac{1}{\gamma} \frac{d}{d t^{\prime}}\left(\gamma \lambda_{m} T_{B}\right)\right] d t^{\prime} .
$$

Легко проверить, что (55) можно представить как

$$
\varphi_{1}=-\frac{4}{m}\left(\gamma \lambda_{m} T_{B} \mathscr{R}^{2}\right)+\int_{\tau}^{t} \frac{4 \gamma \lambda_{m} T_{B}}{m} \frac{d}{d t^{\prime}}\left(\mathscr{R}^{2}\right) d t^{\prime}-C_{1},
$$

где $C_{1}-$ константа интегрирования.

После этого проинтегрируем обе части уравнения (54) по времени от $\tau$ до некоторого момента $t$. Тогда имеем

$$
\begin{aligned}
& \gamma^{2} \mathscr{R}^{3}\left(\frac{d^{2} \mathscr{R}}{d t^{2}}+\frac{1}{\gamma} \frac{d \gamma}{d t} \frac{d \mathscr{R}}{d t}+\frac{4 \lambda_{m} T_{B}}{m \gamma \mathscr{R}}\right)+C_{1} \\
& =\int_{\tau}^{t} d t^{\prime}\left[\frac { 4 \mathscr { R } ^ { 2 } \gamma } { m } \left\{\frac{\lambda_{m} T_{B}}{\mathscr{R}^{2}} \frac{d}{d t^{\prime}}\left(\mathscr{R}^{2}\right)+\Lambda_{\beta 0} \frac{d}{d t}\left[\ln \left(\frac{\lambda_{m} T_{B}}{\Lambda_{\beta 0}}\right)\right]\right.\right. \\
& \left.\left.+\int \chi_{b} S d \mathbf{r}_{\perp}+\frac{\tilde{k}_{S}^{2}}{2 \gamma} \frac{d}{d t}\left(\gamma \lambda_{m} T_{B}\left(D^{*}\right)^{2}\right)\right\}\right]
\end{aligned}
$$


Рассмотрим два слагаемых, стоящих под интегралом в правой части последнего уравнения:

$$
\varphi_{2} \equiv \frac{\lambda_{m} T_{B}}{\mathscr{R}^{2}} \frac{d}{d t^{\prime}}\left(\mathscr{R}^{2}\right)+\Lambda_{\beta 0} \frac{d}{d t}\left[\ln \left(\frac{\lambda_{m} T_{B}}{\Lambda_{\beta 0}}\right)\right] .
$$

Тогда после простых преобразований имеем

$$
\varphi_{2}=\frac{\lambda_{m} T_{B}}{\mathscr{R}^{2}} \frac{d}{d t^{\prime}}\left(\mathscr{R}^{2}\right)+\frac{\Lambda_{\beta 0}}{\lambda_{m} T_{B}} \frac{d\left(\lambda_{m} T_{B}\right)}{d t^{\prime}}-\frac{d}{d t^{\prime}} \Lambda_{\beta 0} .
$$

Далее рассмотрим величину

$$
\tilde{\Gamma}=\frac{\Lambda_{\beta 0}}{\lambda_{m} T_{B}}-\ln \left(\frac{\mathscr{R}^{2}}{2 R_{c}^{2}}\right)
$$

В этом случае находим

$$
\begin{aligned}
-\lambda_{m} T_{B} \frac{d \tilde{\Gamma}}{d t^{\prime}}= & -\lambda_{m} T_{B} \frac{d}{d t^{\prime}}\left[\frac{\Lambda_{\beta 0}}{\lambda_{m} T_{B}}-\ln \left(\frac{\mathscr{R}^{2}}{2 R_{c}^{2}}\right)\right]=-\frac{d}{d t^{\prime}} \Lambda_{\beta 0} \\
& +\frac{\Lambda_{\beta 0}}{\lambda_{m} T_{B}} \frac{d\left(\lambda_{m} T_{B}\right)}{d t^{\prime}}+\frac{\lambda_{m} T_{B}}{\mathscr{R}^{2}} \frac{d}{d t^{\prime}}\left(\mathscr{R}^{2}\right) .
\end{aligned}
$$

Сравнивая правые части (59) и (61), имеем

$$
\varphi_{2}=-\lambda_{m} T_{B} \frac{d \tilde{\Gamma}}{d t^{\prime}}
$$

Подставляя далее (62) и (58) в уравнение (57), окончательно находим

$$
\begin{aligned}
\frac{d^{2} \mathscr{R}}{d t^{2}} & +\frac{1}{\gamma} \frac{d \gamma}{d t} \frac{d \mathscr{R}}{d t}+\frac{4 \lambda_{m} T_{B}}{m \gamma \mathscr{R}}=\frac{1}{\gamma^{2} \mathscr{R}^{3}} \int_{\tau}^{t} d t^{\prime} \\
& \times\left[\frac { 4 \mathscr { R } ^ { 2 } \gamma } { m } \left\{\int \chi_{b} S d \mathbf{r}_{\perp}+\frac{\tilde{k}_{S}^{2}}{2 \gamma} \frac{d}{d t}\left(\gamma \lambda_{m} T_{B}\left(D^{*}\right)^{2}\right)\right.\right. \\
& \left.\left.-\lambda_{m} T_{B} \frac{d \tilde{\Gamma}}{d t^{\prime}}\right\}\right]+\frac{K_{0}}{\gamma^{2} \mathscr{R}^{3}},
\end{aligned}
$$

где $K_{0} \equiv-C_{1}$.

Полученное уравнение (63) представляет собой обобщение уравнения для среднеквадратичного радиуса пучка на случай развития РШН РЭП в рамках линейной стадии неустойчивости.

На рис. 1 для случая отсутствия энергетических потерь $(d \gamma / d t=0)$ представлены полученные с помощью (63) зависимости безразмерного среднеквадратичного радиуса $\mathscr{R}_{1}=\mathscr{R} / R_{b 0}$ от безразмерной дистанции распространения $z_{1}=z k_{S}$ для случаев $D_{1}^{*}=0$ (кривая 1$)$ и $\left(D_{1}^{*}\right)^{2}=2 \sin ^{2}\left(\Omega_{1} z_{1}\right)$ (кривая 2). Здесь $R_{b 0}-$ начальный характерный радиус пучка, $k_{S}-$ характерное бетатронное волновое число для электронов РЭП, $\Omega_{1}$ - заданная безразмерная пространственная частота изменения величины $D_{1}^{*}$. При получении графиков на рис. 1 были взяты следующие параметры: $\lambda_{m}=0.8, K_{1}=K_{0} /\left(\gamma \beta c R_{b 0}^{2} k_{S}\right)^{2}=1.5, \Omega_{1}=1$, $\left.\mathscr{R}_{1}\right|_{z_{1}=0}=1, d \mathscr{R}_{1} /\left.d z_{1}\right|_{z_{1}=0}=0.3$.

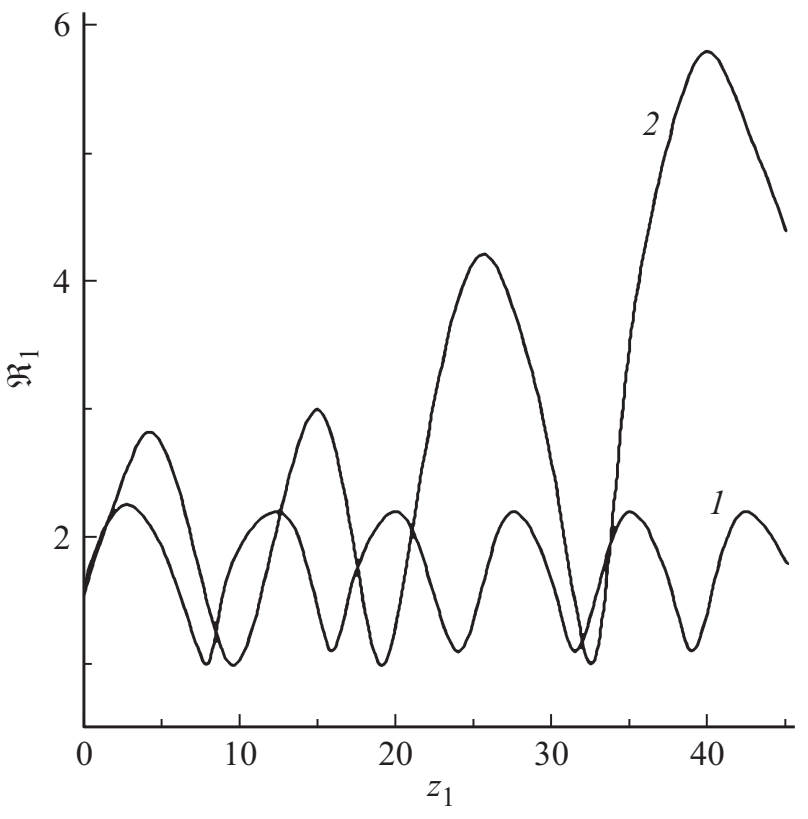

Рис. 1.

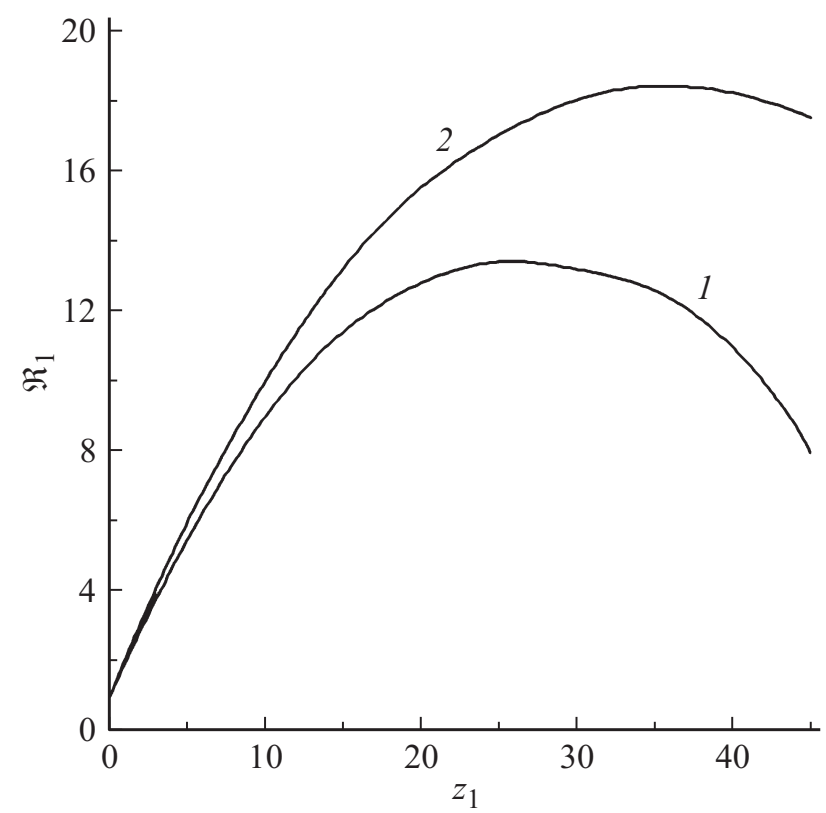

Рис. 2.

На рис. 2. представлены те же кривые, но при более слабом пинч-потенциале, когда $\lambda_{m}=0.4$, и более высоком эмиттансе $K_{1}=1$.

Из рис. 1 и 2 следует, что полученное уравнение огибающей демонстрирует существенное увеличение среднеквадратичного радиуса за счет дефокусировки РЭП при развитии резистивной шланговой неустойчивости пучка.

Кроме того, для квазистационарного пучка нетрудно получить обобщение уравнения Нордсика на рассматриваемый случай развития РШН пучка. В этой ситуации 
уравнение Нордсика принимает вид

$$
\begin{aligned}
\frac{d}{d t}\left[\ln \left(\gamma \mathscr{R}^{2} \lambda_{m} T_{B}\right)\right]= & \frac{1}{\lambda_{m} T_{B}} \int \chi_{b} S d \mathbf{r}_{\perp}-\frac{d \tilde{\Gamma}}{d t} \\
& +\frac{1}{\lambda_{m} T_{B}} \frac{\tilde{k}_{S}^{2}}{2 \gamma} \frac{d}{d t}\left(\gamma \lambda_{m} T_{B}\left(D^{*}\right)^{2}\right) .
\end{aligned}
$$

Данное уравнение отличается от известного уравнения Нордсика двумя последними членами в правой части, которые характеризуют соответственно эффект фазового перемешивания траекторий частиц и процесс потери „пинча“ (самофокусировки) пучка при резистивном запаздывании оси симметрии коллективного электромагнитного поля относительно оси симметрии смещенного в поперечном направлении РЭП.

\section{Заключение}

В настоящей работе с помощью кинетических методов получены уравнения переноса, вириала и динамического равновесия релятивистского электронного пучка, распространяющегося по омическому плазменному канали в случае развития резистивной шланговой неустойчивости. Получено уравнение огибающей пучка, испытывающего указанную неустойчивость.

Работа выполнена в рамках Тематического плана фундаментальных НИР, выполняемых СПбГУ по заказу Министерства образования и науки Российской Федерации № 6.0.10.2010.

\section{Список литературы}

[1] Рухадзе А.А., Богданкевич Л.С., Росинский С.Е., Рухлин В.Г. Физика сильноточных релятивистских электронных пучков. М.: Атомиздат, 1980. $167 \mathrm{c.}$

[2] Диденко А.Н., Григорьев В.П., Усов Ю.П. Мощные электронные пучки и их применение. М.: Атомиздат, 1977. $277 \mathrm{c}$.

[3] Кузелев М.В., Рухадзе А.А. Электродинамика плотных электронных пучков в плазме М.: Физматлит, 1990. 336 с.

[4] Миллер $P$. Введение в физику сильноточных пучков заряженных частиц. М.: Мир, 1984. 432 с.

[5] Колесников Е.К., Мануйлов А.С., Филиппов Б.В. Динамика пучков заряженных частиц в газоплазменных средах. СПб.: Изд-во СПбГУ, 2002. 98 с.

[6] Колесников Е.К., Мануйлов А.С., Зеленский А.Г. Динамика релятивистских электронных пучков в режиме ионной фокусировки. Воскресенск: Позитив, 2013. 104 с.

[7] Lee E.P. // Phys. Fluids. 1976. Vol. 19. N 1. P. 60-69.

[8] Колесников Е.К., Мануйлов А.С. // ЖТФ. 2004. Т. 74. Вып. 9. С. 103-107.

[9] Колесников Е.К., Мануйлов А.С. // ЖТФ. 2005. Т. 75. Вып. 7. С. 119-125.

[10] Мануйлов А.С. // ЖТФ. 2005. Т. 75. Вып. 4. С. 103-108.

[11] Lee E.P. // Phys. Fluids. 1978. Vol. 21. N 8. P. 1327-1343.

[12] Надежсдин Е.Р., Сорокин Г.А. // Физика плазмы. 1983. Т. 9. № 5. C. 989-991.
[13] Кондратьев Н.А., Сметанин В.И. // ЖТФ. 2005. Т. 75. Вып. 3. С. 67-73.

[14] Lampe M., Sharp W.M., Hubbard R., Lee E.P. et al. // Phys. Fluids. 1984. Vol. 27. N 12. P. 2921-2936.

[15] Колесников Е.К., Мануйлов А.С. // ЖТФ. 1991. Т. 61. Вып. 12. С. 43-46.

[16] Мануйлов А.С. // ЖТФ. 2013. Т. 83. Вып. 1. С. 80-83.

[17] Колесников Е.К., Мануйлов А.С. // ЖТФ. 2013. Т. 83. Вып. 11. С. 153-155. 